\title{
HEMORRAGIA INTRAVENTRICULAR Y LEUCOMALACIA PERIVENTRICULAR EN NEONATOS DE BAJO PESO AL NACER EN TRES HOSPITALES DE LIMA, PERÚ
}

\author{
Alonso Zea-Vera ${ }^{1, a}$, Christie Gloria Turín ${ }^{1, a}$, María Susana Rueda1,a; Daniel Guillén-Pinto ${ }^{2, b}$, Pilar Medina-Alva ${ }^{3, b}$, \\ Alfredo Tori ${ }^{4, b}$, María Rivas ${ }^{5, b}$, Jaime Zegarra ${ }^{2, c}$, Anne Castañeda ${ }^{4, c}$, Luis Cam ${ }^{6, c}$, Theresa J. Ochoa ${ }^{7, d}$
}

\begin{abstract}
RESUMEN
Con el objetivo de describir la frecuencia y severidad de la hemorragia intraventricular y leucomalacia periventricular en neonatos de bajo peso en tres hospitales de Lima, Perú se evaluaron 385 neonatos menores de $2000 \mathrm{~g}$ de peso al nacer durante mayo del 2012 a julio del 2014. Se obtuvo ultrasonidos cerebrales a las 40 semanas de gestación, 3-5 días de vida y 3-4 semanas de vida. Hemorragia intraventricular se presentó en 19,2\% neonatos con menos de $1500 \mathrm{~g}$ y fue severa (grado III o con infarto hemorrágico periventricular) en 9,6\% neonatos menores de $1500 \mathrm{~g}$. La mortalidad en neonatos con hemorragia intraventricular fue de $47,1 \%$, se encontró leucomalacia periventricular en $5,4 \%$ de los neonatos menores de $1500 \mathrm{~g}$. Ambos diagnósticos fueron más frecuentes en neonatos con menor peso. La frecuencia de hemorragia intraventricular es similar a lo reportado en otros países; sin embargo, la severidad y mortalidad es mayor.

Palabras clave: Hemorragia cerebral intraventricular; Leucomalacia periventricular; Ultrasonografía. (Fuente: DeCS BIREME).

\section{INTRAVENTRICULAR HEMORRHAGE AND PERIVENTRICULAR LEUKOMALACIA IN LOW BIRTH-WEIGHT NEONATES IN THREE HOSPITALS IN LIMA, PERU}

\begin{abstract}
To describe the frequency and severity of intraventricular hemorrhage and periventricular leukomalacia in low birth-weight neonates in three hospitals in Lima, Peru, 385 newborn babies weighing under $2,000 \mathrm{~g}$ at birth were evaluated between May 2012 and July 2014. Brain ultrasounds were obtained at 40 weeks' gestation, 3-5 days of life, and 3-4 weeks of life. Intraventricular hemorrhage occurred in $19.2 \%$ of neonates weighing under $1,500 \mathrm{~g}$ and was severe (grade III or with periventricular hemorrhagic infarction) in $9.6 \%$ of neonates under $1,500 \mathrm{~g}$. Mortality in infants with intraventricular hemorrhage was $47.1 \%$, while periventricular leukomalacia was found in $5.4 \%$ of neonates $1,500 \mathrm{~g}$ and under; both diagnoses were more frequent in lower-weight babies. The frequency of intraventricular hemorrhage is similar to that reported in other countries; however, severity and mortality are greater.
\end{abstract}

Keywords: Intraventricular hemorrhage; Periventricular leukomalacia; Brain ultrasound. (source: MeSH NLM).

\section{INTRODUCCIÓN}

La hemorragia intraventricular y la leucomalacia periventricular (LPV) son frecuentes en el periodo neonatal, especialmente en prematuros, y están asociadas a la parálisis cerebral y retraso del desarrollo (1). A pesar que la incidencia de hemorragia intraventricular y LPV ha disminuido en países desarrollados, continúan siendo un problema significativo debido a que más prematuros están sobreviviendo el periodo neonatal (2). La supervivencia de prematuros también ha aumentado en Latinoamérica ${ }^{(3,4)}$, lamentablemente, los estudios en esta región son pocos y usualmente están basados en revisiones retrospectivas de historias clínicas.

\footnotetext{
Instituto de Medicina Tropical “Alexander von Humboldt”, Universidad Peruana Cayetano Heredia. Lima, Perú.

Hospital Nacional Cayetano Heredia. Lima, Perú.

Instituto Nacional Materno Perinatal. Lima, Perú.

Hospital Nacional Guillermo Almenara Irigoyen. Lima, Perú.

Hospital Nacional Madre Niño San Bartolomé. Lima, Perú.

Hospital Nacional Alberto Sabogal Sologuren. Lima, Perú.

University of Texas School of Public Health. Houston, Tx, USA.

Médico cirujano; ${ }^{\mathrm{b}}$ médico neurólogo pediatra; ${ }^{\mathrm{c}}$ médico neonatólogo; ${ }^{\mathrm{d}}$ médico infectólogo pediatra

Recibido: 11/09/2018 Aprobado: 06/06/2019 En línea: 13/08/2019
}

Citar como: Zea-Vera A, Turín CG, Rueda MS, Guillén-Pinto D, Medina-Alva P, Tori A, et al. Hemorragia intraventricular y leucomalacia periventricular en neonatos de bajo peso al nacer en tres hospitales de Lima, Perú. Rev Peru Med Exp Salud Publica. 2019;36(3):448-53. doi:http://dx.doi.org/10.17843/rpmesp.2019.363.3922. 
El ultrasonido (US) cerebral es un método rápido, económico, y sensible para detectar hemorragia intraventricular y LPV ${ }^{(5)}$. Muchos pacientes con hemorragia intraventricular y LPV no tienen hallazgos clínicos en el periodo neonatal, lo que hace difícil diagnosticarlo si no se tiene un programa de tamizaje (1). La Academia Americana de Neurología y la Sociedad de Neurología Pediátrica de Estados Unidos recomienda que todos los neonatos menores de 30 semanas de edad gestacional (EG) deben tener un US inclusive si no tienen síntomas ${ }^{(5)}$. El objetivo de este estudio es describir la frecuencia y severidad de hemorragia intraventricular y LPV detectada por US en neonatos de bajo peso al nacer en tres hospitales de Lima, Perú usando un protocolo de tamizaje estandarizado.

\section{EL ESTUDIO}

Se realizó un análisis secundario del ensayo clínico «Lactoferrina para prevención de sepsis en infantes» (NEOLACTO, ClinicalTrials.gov: NCT01525316). Fueron incluidos los neonatos en tres unidades de cuidados intensivos neonatales (UCIN) de Lima, Perú (Hospital Nacional Cayetano Heredia, Hospital Nacional Guillermo Almenara, Hospital Nacional Alberto Sabogal) con un peso al nacer entre 500 a $2000 \mathrm{~g}$. Todos los pacientes fueron enrolados antes de las 72 horas de vida. Los neonatos con problemas gastrointestinales que eviten utilizar la vía enteral, condiciones genéticas que afecten el desarrollo significativamente, historia familiar de alergia a la proteína de leche de vaca y dificultad de completar un seguimiento de dos años fueron excluidos. El enrolamiento se realizó de mayo del 2012 a julio del 2014 y todos los pacientes fueron seguidos hasta los dos años de edad corregida, pero para este estudio se usaron los datos hasta el alta del hospital.

Se realizaron US cerebrales a todos los neonatos como parte del estudio. Los US se realizaron a los 3-5 días de vida, 3-4 semanas de vida y a las 40 semanas de EG. $\mathrm{Si}$ el segundo y tercer US estaban planeados con menos de dos semanas de separación sólo se realizó el US a las 40 semanas de EG. Todos los pacientes con al menos un US fueron incluidos en este análisis. La información prenatal y perinatal se obtuvo al momento del enrolamiento.

Todos los US fueron realizados e interpretados por uno de los neurólogos pediatras involucrados en el estudio (DG, AT, PM). Los equipos que se usaron fueron Sonosite ${ }^{\circledR}$ Titan con un transductor de $5-10 \mathrm{~Hz}$ y Sonoscape ${ }^{\circledR} 6$ con un transductor de $10 \mathrm{~Hz}$. La hemorragia intraventricular se clasificó de acuerdo a la clasificación de Papile et al. modificada por Volpe ${ }^{(1)}$. Si se encontraron diferentes grados de hemorragia intraventricular en diferentes evaluaciones, se utilizó el mayor grado para el análisis. La LPV fue

\section{MENSAJES CLAVE}

Motivación para realizar el estudio. No se conoce con exactitud la frecuencia de hemorragia intraventricular y leucomalacia periventricular en el Perú.

Principales hallazgos. Nuestro estudio sugiere una mejora en la frecuencia de estas patologías, sin embargo, la severidad de esta sigue siendo elevada.

Implicancias. Se necesitan desarrollar estrategias para combatir la alta tasa de mortalidad en hemorragia intraventricular.

definida como la alteración persistente de la sustancia blanca periventricular. Otros hallazgos reportados incluyen ventriculomegalia, hemorragia intraparenquimal y edema sugerente de isquemia cerebral. Se consideró grado I y II como hemorragia intraventricular leve y el grado III e infarto hemorrágico periventricular (IHP) como hemorragia intraventricular severa.

La frecuencia de cada uno de los hallazgos en US fue analizada. Se inspeccionaron los histogramas y se utilizó el método de Shapiro-Wilk para evaluar la normalidad de las variables cuantitativas. Utilizamos proporciones para describir las variables cualitativas y media y desviación estándar para describir las variables cuantitativas. Si una variable cuantitativa no tiene una distribución normal se usó la mediana y rango inter-cuartil. El análisis se realizó utilizando Stata $11^{\circledR}$.

El ensayo clínico NEOLACTO ha sido aprobado por el comité institucional de ética de la Universidad Peruana Cayetano Heredia, la Universidad de Texas en Houston y cada uno de los hospitales participantes. Los padres de todos los participantes firmaron un consentimiento informado. Todos los datos se almacenaron de forma confidencial en el servidor de la Universidad Peruana Cayetano Heredia. Sólo los investigadores tuvieron acceso a los datos. Para este estudio los investigadores sólo accedieron a la base de datos sin identificadores. No se accedieron las historias clínicas o fuentes primarias de información de los pacientes.

\section{HALLAZGOS}

Se enrolaron 414 neonatos en el ensayo clínico, y en $385(93,0 \%)$ se realizó al menos un US cerebral. Seis pacientes murieron antes del primer US, cinco pacientes fueron retirados del estudio antes del primer US y en 18 pacientes no se pudieron realizar por otras razones. Las características de los pacientes incluidos en este estudio fueron similares a la cohorte total (Tabla 1). $239(62,1 \%)$ tuvieron un peso al nacer menor de $1500 \mathrm{~g}$ y 164 (42,6\%) nacieron a las 30 semanas o menos de EG. 
Tabla 1. Características de la cohorte de neonatos que ingresaron al estudio en tres hospitales de Lima, Perú

\begin{tabular}{|c|c|c|}
\hline \multirow{2}{*}{ Variable } & $\begin{array}{c}\text { Neonatos } \\
\text { Incluidos }=\mathbf{3 8 5}\end{array}$ & $\begin{array}{c}\text { Cohorte Total } \\
=414\end{array}$ \\
\hline & n (\%) & n (\%) \\
\hline Peso al nacer en gramos* & $1381,1(355,9)$ & $1380,1(362,0)$ \\
\hline Edad gestacional* $^{\star}$ & $30,8(2,9)$ & $30,8(3,0)$ \\
\hline Sexo masculino & $216(56,1)$ & $230(55,6)$ \\
\hline Apgar a los 5 minutos* & $8,4(1,2)$ & $6,9(2,0)$ \\
\hline Cesárea & $313(81,3)$ & $332(80,4)$ \\
\hline Preeclampsia & $117(30,4)$ & $122(29,5)$ \\
\hline Esteroides antenatales & $271(70,39)$ & $293(70,7)$ \\
\hline Infección periparto & $99(25,7)$ & $104(25,1)$ \\
\hline $\begin{array}{l}\text { Duración de hospitalización } \\
\text { en días * }\end{array}$ & $33,1(24,0)$ & $32,4(24,1)$ \\
\hline
\end{tabular}

* media (desviación estándar)

Se realizaron 376 US a los 3-5 días de vida (en 9 pacientes no se pudo realizar), 316 US a las 3-4 semanas de vida (32 murieron o se retiraron del estudio antes de esta edad, 30 no requirieron US por protocolo, y en 7 el US no pudo realizarse), y 321 a las 40 semanas de EG (45 murieron o se retiraron antes de esta edad, y en 19 no se pudo realizar).

Se diagnosticó hemorragia intraventricular, con o sin infarto hemorrágico periventricular (IHP), en $53(13,8 \%)$ de todos los neonatos y en $46(19,2 \%)$ de los neonatos con un peso al nacer menor de $1500 \mathrm{~g}$. De todos los pacientes con hemorragia intraventricular, $16(30,2 \%)$ tuvieron grado I sin IHP, 13 (24,5\%) tuvieron grado II sin IHP, y 12 $(22,6 \%)$ tuvieron grado III sin IHP. IHP se diagnosticó en 12 (22,6\%); 11 con hemorragia intraventricular grado III y 1 con hemorragia intraventricular grado II (Tabla 2).
Se encontró LPV en $14(3,6 \%)$ de la población. Esta fue más frecuente en neonatos con menor peso al nacer y sólo estuvo presente en uno de los neonatos con un peso al nacer mayor que $1500 \mathrm{~g}$. Se encontró ventriculomegalia en siete $(1,8 \%)$ de los pacientes. La mayoría fue consecuencia de hemorragia intraventricular y sólo un caso fue congénito. Hallazgos sugerentes de hemorragia parenquimal o isquemia cerebral se encontraron en $5(1,3 \%)$ y $8(2,1 \%)$ respectivamente (Tabla 2 ).

El peso al nacer se relacionó inversamente con la severidad de hemorragia intraventricular. De los 53 casos de hemorragia intraventricular, 47 (88,7\%) fueron diagnosticados en el primer US (entre los 3-5 días de vida). En 45 pacientes el máximo grado de hemorragia intraventricular se vio en el primer US y en dos pacientes la hemorragia intraventricular progresó en el segundo US. Seis $(11,3 \%)$ de los casos de hemorragia intraventricular fueron diagnosticados por primera vez en el segundo US (3 grado I y 3 grado II).

El mayor grado de hemorragia intraventricular y la presencia de IHP estuvo asociado a mayor mortalidad. La ventriculomegalia fue más común en pacientes con hemorragia intraventricular (Tabla 3).

\section{DISCUSIÓN}

Se encontró hemorragia intraventricular en 19,2\% neonatos con menos de $1500 \mathrm{~g}$ de peso al nacer y fue severa (grado III o con infarto hemorrágico periventricular) en $9,6 \%$ de estos. La mortalidad en neonatos con hemorragia intraventricular fue de 47,1\%. Se encontró LPV en 5,4\% de los neonatos menores de $1500 \mathrm{~g}$. Ambos diagnósticos fueron más frecuentes en neonatos con menor peso.

Tabla 2. Hallazgos en el ultrasonido cerebral según peso de los neonatos en tres hospitales de Lima, Perú

\begin{tabular}{|c|c|c|c|c|}
\hline \multirow{2}{*}{ Variables } & $<1001 \mathrm{~g}(\mathrm{~N}=69)$ & $1001-1500 \mathrm{~g}(\mathrm{~N}=170)$ & $>1500 \mathrm{~g}(\mathrm{~N}=146)$ & Total $(\mathrm{N}=385)$ \\
\hline & n (\%) & n (\%) & n (\%) & n (\%) \\
\hline Ultrasonido normal & $40(58,0)$ & $139(81,8)$ & $134(91,8)$ & $313(81,3)$ \\
\hline \multicolumn{5}{|l|}{ Grados de hemorragia intraventricular } \\
\hline Grado I* & $5(7,2)$ & $7(4,1)$ & $4(2,7)$ & $16(4,2)$ \\
\hline Grado II* & $6(8,7)$ & $5(2,9)$ & $2(1,4)$ & $13(3,4)$ \\
\hline Grado III* & $5(7,2)$ & $6(3,5)$ & $1(0,7)$ & $12(3,1 \%)$ \\
\hline Infarto hemorrágico periventricular & $8(11,6)$ & $4(2,4)$ & $0(0,0)$ & $12(3,1)$ \\
\hline Ventriculomegalia & $2(2,9)$ & $3(1,8)$ & $2(1,4)$ & $7(1,8)$ \\
\hline Leucomalacia periventricular & $5(7,3)$ & $8(4,7)$ & $1(0,7)$ & $14(3,6)$ \\
\hline Isquemia cerebral & $1(1,5)$ & $3(1,8)$ & $4(2,7)$ & $8(2,1)$ \\
\hline Hemorragia intraparenquimal & $0(0,0)$ & $4(2,4)$ & $1(0,7)$ & $5(1,3)$ \\
\hline
\end{tabular}

* Sin infarto hemorrágico periventricular 
Tabla 3. Consecuencias de la hemorragia intraventricular en neonatos de tres hospitales de Lima, Perú

\begin{tabular}{|c|c|c|c|}
\hline \multirow{2}{*}{ Consecuencia } & Mortalidad & Ventriculomegalia* & Total \\
\hline & n (\%) & n (\%) & N (\%) \\
\hline Sin hemorragia intraventricular & $29(8,7)$ & $3(1,0)$ & 332 \\
\hline \multicolumn{4}{|l|}{ Grados de hemorragia intraventricular } \\
\hline Grado I & $6(37,5)$ & $0(0,0)$ & 16 \\
\hline Grado II & $4(30,7)$ & $1(11,1)$ & 13 \\
\hline Grado III & $5(40,6)$ & $1(14,3)$ & 12 \\
\hline Infarto Hemorrágico Periventricular & $10(83,3)$ & $0(0,0)$ & 12 \\
\hline
\end{tabular}

* Porcentajes calculados en sobrevivientes.

La frecuencia de hemorragia intraventricular en nuestro estudio fue de $13,8 \%$ para toda la cohorte y $19,3 \%$ para los nacidos con peso menor de $1500 \mathrm{~g}$. La frecuencia de estas condiciones en dos de los hospitales incluidos en este estudio ha sido estudiada anteriormente en hospitales de Lima, Perú (6-8); de 1999 al 2001 la incidencia de hemorragia intraventricular en neonatos nacidos antes de las 34 semanas de EG en el Hospital Nacional Cayetano Heredia fue $44,4 \%$ (7) y durante 2004-2005 la incidencia de hemorragia intraventricular en todos los pacientes de UCIN, no solo prematuros, fue de $20 \%{ }^{(6)}$. Un estudio realizado en el Hospital Nacional Guillermo Almenara reportó una incidencia de $33,5 \%$ en neonatos con un peso al nacer menor de $1500 \mathrm{~g}$ entre 2010 a $2012^{(8)}$.

Se debe considerar que el acceso y la potencia de las máquinas de US son mayores actualmente; además, la EG promedio de la cohorte estudiada es menor que la EG promedio en los estudios citados. A pesar que es difícil comparar los resultados con estudios previos, se esperaría que estos factores resulten en un aumento de la frecuencia de hemorragia intraventricular en nuestra cohorte; sin embargo, encontramos lo contrario. Esto sugiere una reducción en la frecuencia de hemorragia intraventricular en los hospitales evaluados.

Otros países en Latinoamérica han reportado mejoras similares. La incidencia de hemorragia intraventricular en Brasil en neonatos con un peso al nacer menor de $1500 \mathrm{~g}$ se redujo de $50 \%$ en 1991 a $11 \%$ en el $2005^{(9)}$. La incidencia de hemorragia intraventricular en nuestra cohorte $(19,3 \%)$ es similar a un reporte de dos hospitales de Colombia de 1999-2004 (10,11). En Estados Unidos también se ha visto una reducción de hemorragia intraventricular, de $40 \%$ en los años $70^{(12-14)}$, a $15 \%-20 \%$ en los años 80 y $90^{(15-17)}$. Sin embargo, los reportes más recientes han mostrado una incidencia de hemorragia intraventricular de $25 \%{ }^{(1,18)}$, esto debido a la mayor supervivencia de prematuros extremos.

En el presente estudio se diagnosticó hemorragia intraventricular severa (definida como grado III o con IHP) en $45,3 \%$ de los casos de hemorragia intraventricular.
Esto es similar a reportes de Colombia, pero mayor al $30-35 \%$ encontrado en países como Brasil y Estados Unidos ${ }^{(1,10,19,20)}$. El $10 \%$ de los neonatos con peso menor de $1500 \mathrm{~g}$ tuvo hemorragia intraventricular severa, comparado al $7 \%$ en un reporte de múltiples UCIN de Latinoamérica ${ }^{(21)}$ y $6 \%$ en Estados Unidos ${ }^{(22)}$.

La mortalidad encontrada en el presente estudio fue mayor que la reportada en países desarrollados (23), probablemente debido a la mayor cantidad de hemorragia intraventricular severa en la cohorte estudiada. Se diagnosticó ventriculomegalia posthemorrágica en sólo $7,7 \%$ de pacientes, una mejoría comparada a $42,6 \%$ reportada en el Hospital Cayetano Heredia de 1999 a $2001^{(7)}$. Sin embargo, es difícil comparar estos resultados con países desarrollados ya que es posible que sean bajos debido a la mayor mortalidad en las UCIN del Perú.

En el presente estudio la frecuencia de LPV fue de 5,4\% en neonatos con peso menor de $1500 \mathrm{~g}$. Esto es similar al reporte del Hospital Guillermo Almenara de los años 2004 a $2005{ }^{(6)}$. En Estados Unidos la frecuencia de LPV en pacientes menores de $1500 \mathrm{~g}$ fue $6 \%$ usando US ${ }^{(24)}$. Sin embargo, comparado a la resonancia magnética, el US sólo tiene $26 \%$ de sensibilidad para detectar LPV no quística ${ }^{(25)}$; por lo tanto, es de esperar que la frecuencia de LPV en la población estudiada sea mayor.

Una de las debilidades del presente estudio es la posibilidad que la intervención del ensayo clínico (suplementos de lactoferrina) haya afectado los resultados. Sin embargo, el $90 \%$ de hemorragia intraventricular ocurren en los primeros tres días de vida, un periodo en el que la mayoría de sujetos no recibieron ninguna intervención. El efecto de la intervención en LPV podría ser más significativo y será parte del análisis principal del ensayo clínico. Otra debilidad es que no se incluyeron todos los neonatos menores de $1500 \mathrm{~g}$ y algunos incluidos no tuvieron US. A pesar de esto, la población estudiada fue similar a las tres unidades neonatales incluidas en el estudio. Las fortalezas del presente estudio incluyen su diseño prospectivo, un seguimiento estandarizado y la inclusión de múltiples hospitales. La mayoría de reportes de países en desarrollo 
son retrospectivos e incluyen un solo hospital, y pueden ser afectados por las prácticas individuales en los hospitales.

En conclusión, los resultados sugieren una disminución en la frecuencia de hemorragia intraventricular en neonatos de muy bajo peso en los hospitales evaluados que está acercándose a los resultados de países desarrollados. Sin embargo, la severidad y mortalidad de pacientes con hemorragia intraventricular sigue siendo mayor. Adicionalmente, con la mejora del cuidado neonatal es esperable que la mayor supervivencia de prematuros extremos resulte en mayor incidencia de hemorragia intraventricular y LPV. Es importante evaluar los factores asociados a mayor severidad y mortalidad para generar estrategias para combatirlas.

Agradecimientos: Agradecemos a todos los miembros del grupo de investigación NEOLACTO: Rospigliosi M, MD, Borda G, MD,
Webb V, MD, Lino A, MD, Cama A, MD, Llanos R, MD, Chumbes O, MD, Cuba, L, MD, Tresierra J, MD, Chincaro C, MD, Alarcon W, MD, Bravo E, MD, Pacheco K, MD, Campos M, MD, Tucto L, RN, Suarez C, RN, Huanay M, RN, Rojas N, RN. También queremos expresar nuestra más sincera gratitud a las madres de nuestros pacientes por su participación y compromiso con el proyecto.

Contribuciones de los autores: AZV y TJO participaron en la concepción y diseño del artículo. AZV, CGT, MSR, DGO, PMA, AT, MR, participaron en la recolección de datos. JZ, AC, LC participaron en el enrolamiento de pacientes y recolección de datos. AZV participó en el análisis e interpretación de datos y redacción del artículo. Todos los autores revisaron y aprobaron el artículo.

Fuentes de Financiamiento: Este estudio fue financiado por el premio de Public Health Service (R01-HD067694) del National Institute of Child Health and Human Development (NICHD), USA.

Conflicto de Interés: Ninguno de los autores reporta conflictos de interés.

\section{REFERENCIAS BIBLIOGRÁFICAS}

1. Volpe JJ. Neurology of the newborn. Philadelphia: Saunders/Elsevier; 2008.

el Hospital Nacional Cayetano Heredia Lima. Rev Peru Pediatr. 2005;58(1):4-11.

2. Osterman MJK, Kochanek KD,8. Lizama O, Hernández H, Rivera F, MacDorman MF, Strobino DM, Guyer B. Annual summary of vital statistics: 20122013. Pediatrics. 2015;135(6):1115-25.

3. Beck S, Wojdyla D, Say L, Betran AP, Merialdi M, Requejo JH, et al. The worldwide incidence of preterm9. birth: a systematic review of maternal mortality and morbidity. Bull World Health Organ. 2010;88(1):31-8.

4. Grupo Colaborativo Neocosur. Verylow-birth-weight infant outcomes10. in 11 South American NICUs. J Perinatol. 2002;22(1):2-7.

5. Ment LR, Bada HS, Barnes P, Grant PE, Hirtz D, Papile LA, et al. Practice parameter: neuroimaging of the neonate: report of the Quality11. Standards Subcommittee of the American Academy of Neurology and the Practice Committee of the Child Neurology Society. Neurology. 2002;58(12):1726-38.

6. Medina Alva M del P, Rivera Abbiati F, Antonio A, Montiel Blanco J, Guillen Pinto D. Frecuencias, características y mortalidad asociada a la enfermedad neurológica en la Unidad de Cuidados1 Intensivos Neonatales del Hospital Nacional Cayetano Heredia. Rev Peru Pediatr. 2007;60(1):11-9.

7. Guillen Pinto D, Bellomo Montalvo S. Incidencia, factores asociados y13. Levene MI, Wigglesworth JS, Dubowitz pronóstico de las lesiones cerebrales en prematuros menores de 34 semanas en
Tori A. Incidencia de la hemorragia intraventricular en prematuros de muy bajo peso y sus factores asociados en un hospital nacional de Lima, Perú. Rev Medica Hered. 2014;25(2):1-5.

Marba STM, Caldas JPS, Vinagre LEF, Pessoto MA. Incidence of periventricular/ intraventricular hemorrhage in very low birth weight infants: a 15-year cohort study. J Pediatr (Rio J). 2011;87(6):505-11.

Segovia Morales LO, Latorre Latorre JF, Rodríguez Hernández J, Perez Vera A. Hemorragia intraventricular en niños pretérmino, incidencia y factores de riesgo. Un estudio de corte transversal. MedUNAB. 2003;6(17):57-62.

Ayala Mendoza AM, Carvajal Kalil LF, Carrizosa Moog J, Galindo Hernández Á, Cornejo Ochoa JW, Sánchez Hidalgo Y. Evaluación de la incidencia y los factores de riesgo para hemorragia intraventricular (HIV) en la cohorte de recién nacidos prematuros atendidos en la unidad neonatal del Hospital Universitario San Vicente de Paúl, de Medellín, en el período comprendido. Iatreia. 2007;20(4):341-53.

. Ahmann PA, Lazzara A, Dykes FD, Brann AW, Schwartz JF. Intraventricular hemorrhage in the high-risk preterm infant: incidence and outcome. Ann Neurol. 1980;7(2):118-24.

V. Cerebral structure and intraventricular haemorrhage in the neonate: a real-time ultrasound study. Arch Dis Child. 1981;56(6):416-24.

14. McMenamin JB, Shackelford GD, Volpe JJ. Outcome of neonatal intraventricular hemorrhage with periventricular echodense lesions. Ann Neurol. 1984;15(3):285-90.

15. Philip AG, Allan WC, Tito AM, Wheeler LR. Intraventricular hemorrhage in preterm infants: declining incidence in the 1980s. Pediatrics. 1989;84(5):797-801.

16. Paneth N, Pinto-Martin J, Gardiner J, Wallenstein S, Katsikiotis V, Hegyi T, et al. Incidence and timing of germinal matrix/intraventricular hemorrhage in low birth weight infants. Am J Epidemiol. 1993;137(11):1167-76.

17. Sheth RD. Trends in incidence and severity of intraventricular hemorrhage. J Child Neurol. 1998;13(6):261-4.

18. Hamrick SEG, Miller SP, Leonard C, Glidden DV, Goldstein R, Ramaswamy $\mathrm{V}$, et al. Trends in severe brain injury and neurodevelopmental outcome in premature newborn infants: the role of cystic periventricular leukomalacia. J Pediatr. 2004;145(5):593-9.

19. Mancini MC, Barbosa NE, Banwart D, Silveira S, Guerpelli JL, Leone CR. Intraventricular hemorrhage in very low birth weight infants: associated risk factors and outcome in the neonatal period. Rev Hosp Clin. 1999;54(5):151-4.

20. Neves LAT, Neto C, Antônio J, Kneipp D, Fonseca LG, Rosado MP, et al. 
Hemorragia Intracraniana no recémnascido pré-termo: casuística da UTI Neonatal do Hospital Albert Sabin. HU Rev. 2007;33(2):47-52.

21. Fernández R, D’Apremont I, Domínguez A, Tapia JL, Red Neonatal Neocosur. Survival and morbidity of very low birth weight infant in a South American neonatal network. Arch Argent Pediatr. 2014;112(5):405-12.

22. Horbar JD, Carpenter JH, Badger GJ, Kenny MJ, Soll RF, Morrow $\mathrm{KA}$, et al. Mortality and neonatal morbidity among infants 501 to 1500 grams from 2000 to 2009 . Pediatrics. 2012;129(6):1019-26.
23. Murphy BP, Inder TE, Rooks V, Taylor GA, Anderson NJ, Mogridge N, et al. Posthaemorrhagic ventricular dilatation in the premature infant: natural history and predictors of outcome. Arch Dis Child Fetal Neonatal Ed. 2002;87(1):37-41.

24. Stevenson DK, Wright LL, Lemons JA, Oh W, Korones SB, Papile LA, et al. Very low birth weight outcomes of the National Institute of Child Health and Human Development Neonatal Research Network, January 1993 through December 1994. Am J Obstet Gynecol. 1998;179(6 Pt 1):1632-9.
25. Inder TE, Anderson NJ, Spencer C, Wells S, Volpe JJ. White matter injury in the premature infant: a comparison between serial cranial sonographic and MR findings at term. Am J Neuroradiol. 2003;24(5):805-9.

Correspondencia: Theresa J. Ochoa.

Dirección: Instituto de Medicina Tropical "Alexander von Humboldt", Universidad Peruana Cayetano Heredia, Av. Honorio Delgado 430. San Martin de Porras, Lima 31, Perú, Correo electrónico: Theresa.J.Ochoa@uth.tmc.edu; Theresa. Ochoa@upch.pe.

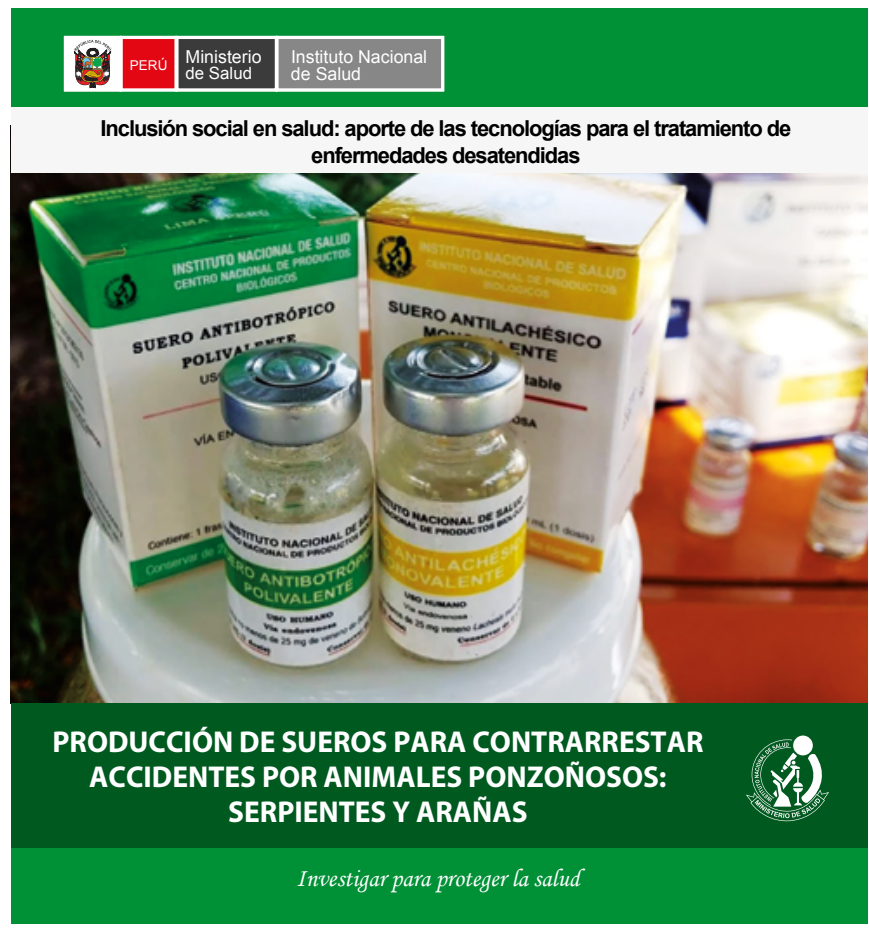

